\title{
HAUSDORFF MATRICES AS BOUNDED OPERATORS OVER $l$
}

\author{
B. E. RHOADES
}

\begin{abstract}
A necessary and sufficient condition is obtained for an arbitrary Hausdorff matrix to belong to $B(l)$. It is then shown that every conservative quasi-Hausdorff matrix is of type $M$.
\end{abstract}

Let $(H, \mu)$ denote the Hausdorff method with generating sequence $\mu=$ $\left\{\mu_{n}\right\}, l=\left\{\left\{x_{n}\right\}\left|\Sigma_{n}\right| x_{n} \mid<\infty\right\}, B(l)$ the algebra of bounded linear operators on $l$. A necessary and sufficient condition is obtained for an arbitrary Hausdorff method to belong to $B(l)$. It is then shown that every conservative quasi-Hausdorff matrix is of type $M$.

An infinite matrix is called triangular if it has only zeros above the main diagonal. Let $B(c)$ denote the algebra of bounded linear operators in $c$, the space of convergent sequences.

LEMMA. Let $A$ be a triangular matrix satisfying:

(1) $A \in B(l)$,

(2) $t_{n}^{*}=\sum_{k=0}^{n}\left|a_{n k}\right|$ is monotone increasing in $n$,

(3) $\lim _{n} t_{n}$ exists, where $t_{n}=\sum_{k=0}^{n} a_{n k}$.

Then $A \in B(c)$.

Proof. Condition (1) is equivalent to $\sup _{k} \sum_{n=k}^{\infty}\left|a_{n k}\right|<\infty$, which implies $\sum_{n=k}^{N}\left|a_{n k}\right|<M$ for every $N>k$, where $M$ is independent of $N$ and $k$. Summing $k$ over $[0, N]$ yields $\sum_{k=0}^{N} \sum_{n=k}^{N}\left|a_{n k}\right| \leqslant M(N+1)$. Interchanging the order of summation gives

$$
\sum_{n=0}^{N} t_{n}^{*} /(N+1) \leqslant M
$$

The left-hand side of (4) is the $N$ th term of the Cesàro transform of order one, $(C, 1)$, of the sequence $\left\{t_{n}^{*}\right\}$. Since $\left\{t_{n}^{*}\right\}$ is monotone increasing, the norm of $A$ in $B(c)$ is $\|A\|_{c}=\lim _{n} t_{n}^{*}$. If $\|A\|_{c}=\infty$, then the total regularity of $(C, 1)$ implies that the 1.h.s. of (4) tends to $\infty$ as $N \rightarrow \infty$, a contradiction. Therefore $\|A\|_{c}$ is finite. Condition (1) implies $A$ has zero column limits and (3) assures the existence of the limit of the row sums. Therefore $A \in B(c)$.

Received by the editors March 21, 1979.

AMS (MOS) subject classifications (1970). Primary 40G05; Secondary $40 C 05$.

Key words and phrases. Hausdorff means, quasi-Hausdorff means, type $M$. 
Theorem 1. Let $H$ be a Hausdorff matrix. Then $H \in B(l)$ if and only if $\mu$ is a moment sequence satisfying

$$
\int_{0}^{1}|d \beta(t)| / t<\infty
$$

Proof. In [3, p. 279] it is shown that, if $\mu$ is a moment sequence, then $H \in B(l)$ if and only if $\mu$ satisfies (5). The theorem in Hardy actually treats conservative quasi-Hausdorff methods $\left(H^{*}, \mu\right)$, but $\left(H^{*}, \mu\right)$ is merely the matrix transpose of $(H, \mu)$, so that the norm condition for the regularity of $H^{*}$ is the same as the norm condition for $H \in B(l)$.

It remains to show that, if $H \in B(l)$, then $\mu$ is a moment sequence.

From [3, p. 254] or [4, Lemma 2, p. 177], $\left\{t_{n}^{*}\right\}$ is monotone increasing in $n$. Each row sum of $H$ adds up to $\mu_{0}$, so (3) of the Lemma is satisfied. Applying the Lemma, $H \in B(c)$, which by [3, p. 260] is equivalent to $\mu$ being a moment sequence.

Let $A \in B(c), A$ a matrix. Then $A$ is said to be of type $M$ if the only solution of $t A=0, t \in l$, is $t=0$.

Theorem 2. Let $\left(H^{*}, \mu\right) \in B(c)$. Then $\left(H^{*}, \mu\right)$ is of type $M$.

In [5] it was shown that $H^{*}$ is of type $M$, provided at most a finite number of the $\mu_{n}$ are zero. We now provide a different proof, which removes that restriction.

Since $H^{*}$ is the matrix transpose of $H$, the condition $H^{*} \in B(c)$ is equivalent to $H \in B(l)$. By Theorem $1, \mu$ is a moment sequence. Thus there exists a function $\beta(t) \in B V[0,1]$ such that $\mu_{n}=\int_{0}^{1} t^{n} d \beta(t)$. Define

$$
F(z)=\int_{0}^{1} t^{z} d \beta(t)
$$

Then $F$ is analytic in $\operatorname{Re} z>0$ and continuous on $\operatorname{Im} z=0$. Moreover, if $b_{i}$ denote the real zeros of $F$ in $\operatorname{Re} z>0$, they satisfy $\Sigma 1 / b_{i}<\infty$, by a result of Carleman [1]. To say that $H^{*}$ is of type $M$ is equivalent to saying that $H$ is 1-1 on $l$.

Since $H=\delta \mu \delta$, where $\delta$ is the triangular matrix of signed binomial coefficients and $\mu$ is a diagonal matrix with diagonal entries $\mu_{n}$, we may use associativity of multiplication and the fact that $\delta$ is its own inverse, to obtain $\mu \delta t=0$; i.e., $\mu_{n} \Delta^{n} t_{0}=0$ for $n=0,1,2, \ldots$ Now appeal to [2, Theorem 1] to conclude that $t$ is a constant sequence. The result follows, since the only constant sequence in $l$ is the zero sequence.

Theorems 1 and 2 are also true for generalized Hausdorff matrices of the form

$$
h_{n k}=\left(\begin{array}{l}
n+\alpha \\
n-k
\end{array}\right) \Delta^{n-k} \mu_{k}, \quad \text { with } \alpha \geqslant 0
$$




\section{REFERENCES}

1. T. Carleman, Über die Approximation analytischer Funktionen durch Aggregate vorgegebener Potenzen, Ark. Mat. 17 (1922), no. 9.

2. W. H. J. Fuchs, $A$ theorem on finite differences with an application to the theory of Hausdorff summability, Proc. Cambridge Philos. Soc. 40 (1944), 188-196.

3. G. H. Hardy, Divergent series, Oxford Univ. Press, London, 1949.

4. A. Jakimovski, B. E. Rhoades and J. Tzimbalario, Hausdorff matrices as bounded operators over $l^{P}$, Math. Z. 138 (1974), 173-181.

5. B. E. Rhoades, Type $M$ for quasi-Hausdorff matrices, Proc. Cambridge Philos. Soc. 68 (1970), 601-604.

Department of Mathematics, Indiana UniverstTy, Bloomington, Indiana 47405 